\title{
Multi-Frequency Modulation Laser Range Finding System
}

\author{
Chen $\mathrm{Yu}^{1}$, Wang Chunyang ${ }^{1}$, Gao Huan ${ }^{2} \&$ Liu Huan ${ }^{1}$ \\ ${ }^{1}$ School of Electronic and Information Engineering, Changchun University of Science and Technology, \\ Changchun, China \\ ${ }^{2}$ School of Science, Changchun University of Science and Technology, Changchun, China \\ Correspondence: Wang Chunyang, School of Electronic and Information Engineering, Changchun University of \\ Science and Technology, Jilin, Changchun, No. 7089 Weixing road, China. Tel: 86-138-4317-6321. E-mail: \\ assma@163.com
}

Received: November 3, 2014

doi:10.5539/mas.v9n4p328

\author{
Accepted: November 15, 2014 Online Published: March 30, 2015 \\ URL: http://dx.doi.org/10.5539/mas.v9n4p328
}

\begin{abstract}
Traditional laser ranging system has a poor phase measuring accuracy, low anti-jamming capability and time-consuming measurement. A multi-frequency modulation laser range finder method is proposed in this paper. System uses phase detection algorithm to calculate the sine of ranging phase for noisy environments, and the angle is calculated by Coordinate Rotation Digital Computer(CORDIC) angle solver algorithm. When the sampling frequency is $500 \mathrm{MHz}$, the word length is 16 -bits, the SNR is $12 \mathrm{~dB}$, the measurement range is $100 \mathrm{~m}$, the phase difference resolution is higher than $0.0213^{\circ}$, and the distance accuracy is $0.10 \mathrm{~mm}$. Experiments proved that the system can meet the need of high-accuracy and low computational complexity.
\end{abstract}

Keywords: laser ranging, multi-frequency modulation, CORDIC, FPGA

\section{Introduction}

At present, laser range finding system is widely used in the areas of nondestructive testing, machine vision and intelligent vehicle cruise, etc [1,2]. Based on the different demands of measurement accuracy and measurement range in different applications, in order to ensure the ranging accuracy and the range of the ranging system, a reasonable frequency combination will be needed. Multi-frequency laser modulation phase range finding system uses multi-frequency to enlarge the range of ranging and improve measurement accuracy, high frequency, high measurement accuracy; low frequency, large measuring range[3-6]. This paper presents a calculation based on the coordinate rotation digital computer angle of the laser phase ranging method. Based on FPGA, a multi frequency laser ranging system has been designed, which has simple hardware structure and the advantages of high ranging accuracy and low computation. The CORDIC is using the basic add and shift operations instead of multiplication, using a series of continuous deflections of the fixed angle to approximate the desired rotation angle.

\section{Laser Range Finding System Design}

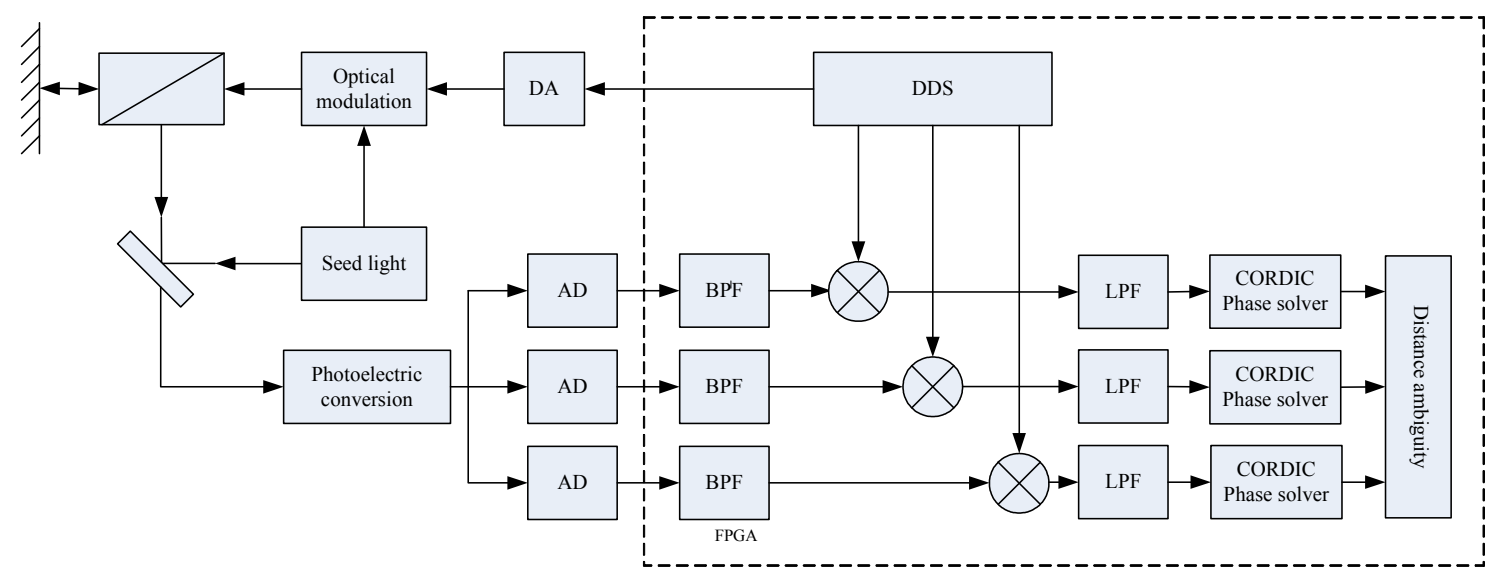

Figure 1 . The theory multi-frequency modulation laser range finding system 
Real time multi-frequency modulation laser range finding system is as shown in Figure 1, the working process is: DDS generates three sine signals of different frequency, after superposition, conversion and then output through DA, the output signal modulates the seed, the modulated signal generates the echo signal through the optical system, then the seed and the echo signal mixing through the lens and become intermediate frequency signal, after photoelectric conversion of the intermediate frequency signal, three signals with phase delay $\Delta \varphi_{1}$, $\Delta \varphi_{2}$ and $\Delta \varphi_{3}$ are generated through the three AD conversion and band pass filter, the sine of signal phase delay can be obtained by mixing the local oscillator, after angle value is calculated by CORDIC operator and transfer to distance solution of fuzzy module, the ranging distance is calculated eventually.

\section{Resolving of the Laser Ranging Signal}

\subsection{Multi-frequency Modulation Real Time Laser Range Finding}

Assuming the emission signal for ranging is:

$$
s(t)=g(t) \cdot \sum_{n=1}^{N} \sin \left(2 \pi f_{n} t+\varphi_{n}\right)
$$

In the equation, $N$ stands for the number of the measuring frequency, $f_{n}$ stands for the frequency of the measuring signal, $\varphi_{n}$ stands for the initial phase, at the same time, $f_{1}>f_{2}>\ldots>f_{N}, g(t)$ stands for the time of the seed. Therefore, the echo signal can be expressed as:

$$
s^{\prime}(t)=A \cdot g(t+\Delta t) \cdot \sum_{n=1}^{N} \sin \left(2 \pi f_{n} t+2 \pi f_{n} \cdot 2 R / c+\phi_{n}+\phi_{o n}+\phi_{i n}\right)
$$

In the equation, $\Delta t$ is the time delay, $A$ is the system-gain, $R$ is the ranging distance, $c$ is the constant speed of light, $\phi_{\text {in }}$ and $\phi_{\text {on }}$ are the phase delays of the input signal and the ranging signal. Assuming $\phi_{\text {in }}+\phi_{\text {on }}=0$, then the signal after mixing can be expressed as:

$$
s^{\prime \prime}(t)=A^{\prime \prime} \sum_{n=1}^{N} \sin \left(2 \pi f_{n} t+2 \pi f_{n} \cdot 2 R / c+\phi_{n}\right)
$$

The normalized signal is:

$$
\overline{s^{\prime \prime}}(t)=\sum_{n=1}^{N} \sin \left(2 \pi f_{n} t+2 \pi f_{n} \cdot 2 R / c+\phi_{n}\right)
$$

After passing through $\mathrm{N}$ band pass filters:

$$
\left\{\begin{array}{l}
s_{1}=\sin \left(2 \pi f_{0} t+2 \pi f_{n} \cdot 2 R / c+\phi_{0}\right) \\
\cdots \\
s_{N}=\sin \left(2 \pi f_{N} t+2 \pi f_{N} \cdot 2 R / c+\phi_{N}\right)
\end{array}\right.
$$

After phase discrimination of the signal in equation (5), the sine of each measuring phase delay can be getting:

$$
\begin{aligned}
& \arcsin \left(\Delta \phi_{n}\right)=\arcsin \left(2 \pi f_{n} \cdot 2 R / c\right) \\
& \Delta \phi_{n}=2 \pi f_{n} \cdot 2 R / c
\end{aligned}
$$

\subsection{Phase Detection Method}

The structure of phase calculating module is shown in Figure 2.

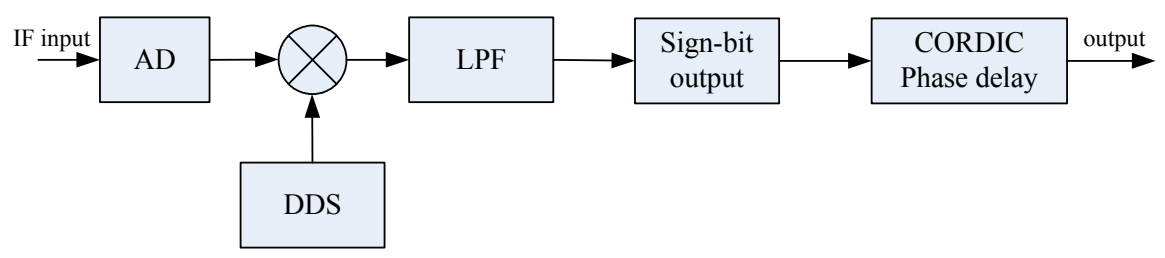

Figure 2. The theory of phase calculation module

Assuming after passing through the frequency selective filter, the signal becomes: 


$$
u(t)=\cos \left(2 \pi f_{c} t\right)
$$

Where $f_{c}$ is the frequency of the measuring sinusoidal signal.

The output signal of DDS is:

$$
L(t)=\sin \left(2 \pi f_{c} t+\Delta \phi\right)
$$

In the equation, $\Delta \phi$ is the phase difference between the input signal and the DDS signal, the multiplier output is:

$$
U(t)=K_{p} \cos 2 \pi f_{c} t \sin \left(2 \pi f_{c}+\Delta \phi\right)
$$

Where $K_{p}$ is the coefficient of the multiplier, after low pass filter, we can get:

$$
U_{l}=\frac{1}{2} K_{p} K_{l} \sin \Delta \phi
$$

Where $K_{l}$ is the coefficient of the low pass filter. After normalization, arcsine $U_{l}, \Delta \phi$ can be getting.

\subsection{Angle Calculating Based On CORDIC}

Coordinate Rotation Digital Computer is using the basic add and shift operations instead of multiplication, using a series of continuous deflections of the fixed angle to approximate the desired rotation angle[7]. In this paper, the system ranging phase delay can be getting by arcsine CORDIC calculation.

$\left(X_{K}, Y_{K}\right)$ is vector $\left(X_{j}, Y_{j}\right)$ rotates $\theta$ :

$$
\begin{aligned}
X_{k} & =R \cos (\beta+\theta) \\
& =R \cos \beta \cdot \cos \theta-R \sin \beta \cdot \sin \theta \\
& =X_{j} \cos \theta-Y_{j} \sin \theta
\end{aligned}
$$

Similar,

$$
Y_{k}=X_{j} \sin \theta+Y_{j} \cos \theta
$$

In matrix way, it can be expressed as:

$$
\begin{aligned}
{\left[\begin{array}{c}
X_{k} \\
Y_{k}
\end{array}\right] } & =\left[\begin{array}{cc}
\cos \theta & -\sin \theta \\
\sin \theta & \cos \theta
\end{array}\right] \cdot\left[\begin{array}{c}
X_{j} \\
Y_{j}
\end{array}\right] \\
& =\cos \theta\left[\begin{array}{cc}
1 & -\tan \theta \\
\tan \theta & 1
\end{array}\right] \cdot\left[\begin{array}{c}
X_{j} \\
Y_{j}
\end{array}\right]
\end{aligned}
$$

Assuming $\theta=\sum_{n=0}^{N} a_{n} \theta_{n}, a_{n}$ is the rotation factor, $a_{n}=1$ while rotating clockwise, $a_{n}=-1$ while rotating counterclockwise, let $\theta_{n}=\arctan \left(2^{-i}\right)$, then $\tan \theta_{n}=2^{-i}, \cos \theta_{n}=\frac{1}{\sqrt{1+2^{-2 i}}}$,

Then:

$$
\left[\begin{array}{c}
X_{k} \\
Y_{k}
\end{array}\right]=\frac{1}{\sqrt{1+2^{-2 i}}}\left[\begin{array}{cc}
1 & -a_{k} 2^{-i} \\
a_{k} 2^{-i} & 1
\end{array}\right] \cdot\left[\begin{array}{c}
X_{k-1} \\
Y_{k-1}
\end{array}\right]
$$

Assuming $Z$ is the sum of the phase accumulation parts after i times rotations (disregard multiplication factor), the equation above can be expressed as:

$$
\left\{\begin{array}{c}
X_{i+1}=X_{i}-a_{i} 2^{-i} Y_{i} \\
Y_{i+1}=Y_{i}+a_{i} 2^{i} X_{i} \\
Z_{i+1}=Z_{i}-a_{i} \arctan 2^{-i}
\end{array}\right.
$$

When $Y_{i}<0, a_{i}=+1$; when $Y_{i}>0, a_{i}=-1$.

After $n$ times of iteration: 


$$
\left\{\begin{array}{c}
X_{n}=A_{n} \sqrt{X_{0}^{2}+Y_{0}^{2}} \\
Y_{n}=0 \\
Z_{n}=Z_{0}+\tan ^{-1}\left(Y_{0} / X_{0}\right)
\end{array}\right.
$$

Where

$$
A_{n}=\prod_{n}\left(1 / \sqrt{1+2^{-2 i}}\right)
$$

\section{Range Finding System Performance Analysis}

Assuming the channel of the range finding system is broadband white noise channel, then the system echo signal is:

The output of the low pass filter is:

$$
U(t)=\cos \left(\omega_{c} t+\Delta \phi\right)+n(t)
$$

$$
U(t)=\frac{1}{2} K_{p} K_{l} \sin \Delta \phi+K_{p}\left[n(t) \cdot \cos \omega_{c} t\right]^{*} h(t)
$$

Let $d(t)=\left(n(t) \bullet \cos \omega_{c} t\right) * h(t), h(t)$ is the system function of the low pass filter.

From equation (11) and (20), the phase arcsine error can be expressed:

$$
e=U(t)-U_{l}=K_{p} d(t)
$$

Assuming the average value $n(t)$ is 0 , gaussian noise with power spectral density $S(\omega)=N_{0} / 2$. Then the physical explanation of the noise item $d(t)$ in equation (25) is as shown in Figure 3:

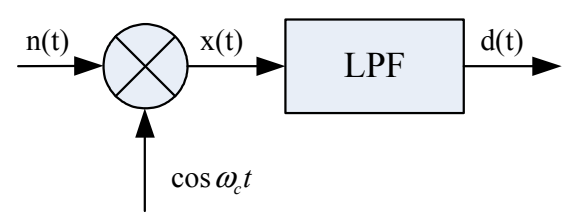

Figure 3. $d(t)$ physical explanation

Let $x(t)=n(t) \cdot \cos \omega_{c} t$, then the autocorrelation function of $x(t)$ is[8]:

$$
\begin{aligned}
R_{x}(\tau) & =\frac{1}{2 \pi} \int_{-\infty}^{\infty} S(\omega)\left[\pi \cdot\left(\delta\left(\omega-\omega_{0}\right)+\delta\left(\omega+\omega_{0}\right)\right] d \omega\right. \\
& =\frac{N_{0}}{4} \int_{-\infty}^{\infty}\left[\delta\left(\omega-\omega_{0}\right)+\delta\left(\omega+\omega_{0}\right)\right] d \omega \\
& =N_{0} / 2
\end{aligned}
$$

The average value $x(t)$ is:

$$
\mu_{x}=E[x(t)]=E\left[n(t) \cdot \cos \omega_{c} t\right]=0
$$

$h(t)$ is low pass filter, assuming its frequency response function is:

$$
H(j \omega)=\frac{1}{1+j \omega R C}
$$

Then,

$$
|H(\omega)|^{2}=\frac{1}{1+(\omega R C)^{2}}
$$

So the power spectral density $S_{d}(\omega)$ of $d_{1}(t)$ is:

$$
S_{d}(\omega)=|H(\omega)|^{2} S_{x}(\omega)=\frac{N_{0} / 2}{1+(\omega R C)^{2}}
$$

The average value of $d(t)$ is: 


$$
\begin{aligned}
\mu_{d} & =E[d(t)] \\
& =E\left[\int_{-\infty}^{\infty} h(t-\tau) x(\tau) d \tau\right] \\
& =\mu_{x} H(0)=0
\end{aligned}
$$

Thus, the average value of $d(t)$ is 0 , the power spectral density is $S_{d}(\omega)=\frac{N_{0} / 2}{1+(\omega R C)^{2}}$, the mathematical expectation of equation (20) is:

$$
\begin{aligned}
\mu & =E(U(t)) \\
& =E\left[K_{p} K_{l} \sin \Delta \phi+K_{p} d(t)\right] \\
& =E\left(\frac{1}{2} K_{p} K_{l} \sin \Delta \phi\right)+K_{p} \cdot E(d(t)) \\
& =\frac{1}{2} K_{p} K_{l} \sin \Delta \phi
\end{aligned}
$$

Then:

$$
\Delta \phi==\arcsin \frac{2 \mu}{K_{p} K_{l}}
$$

Assuming the phase delay is $\pi / 6$, the phase delay error under different SNR can be expressed in Figure 4 .

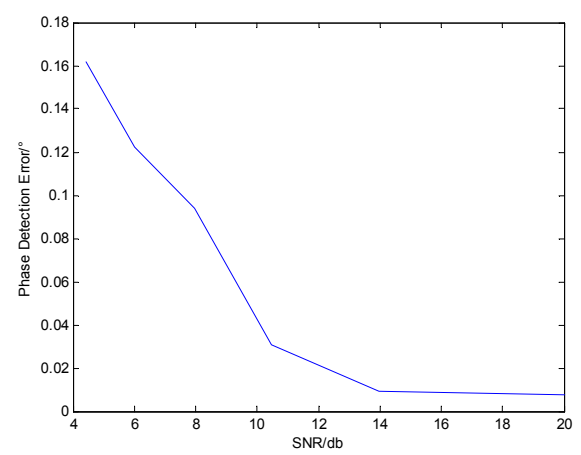

Figure 4. Phase delay error under different SNR

\section{Laser Range Finding Platform Based on FPGA}

In this paper, a laser range finding platform based on FPGA has been developed; the core module of the system adopts the CORDIC IP core of xilinx FPGA. The schematic diagram of Phase detection is as shown in Figure 5.RTL and function simulation diagrams are shown in Figure 6 and Figure 7.

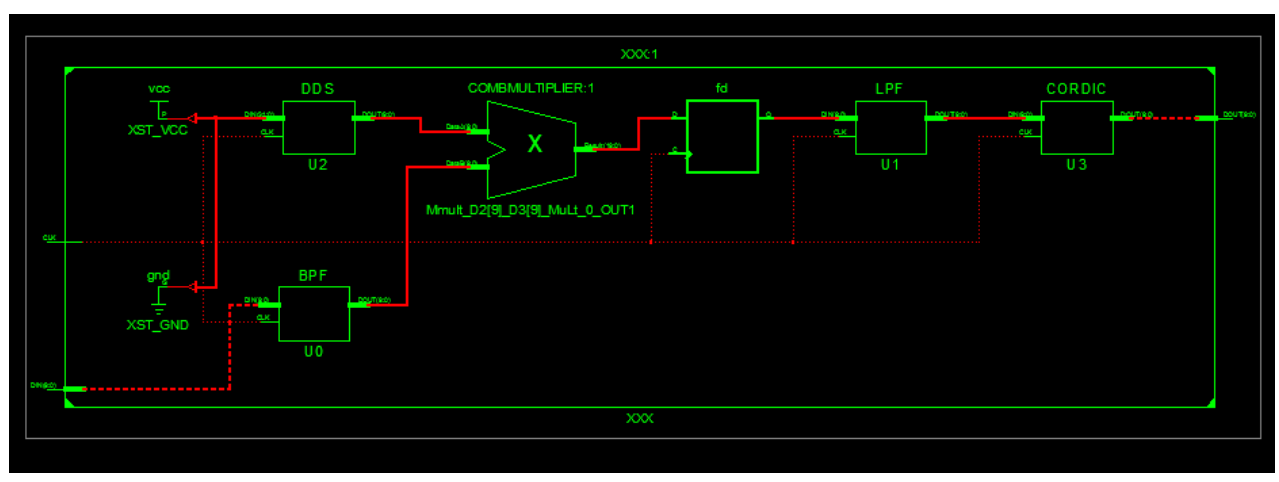

Figure 5. Phase detection algorithm RTL Schematic 


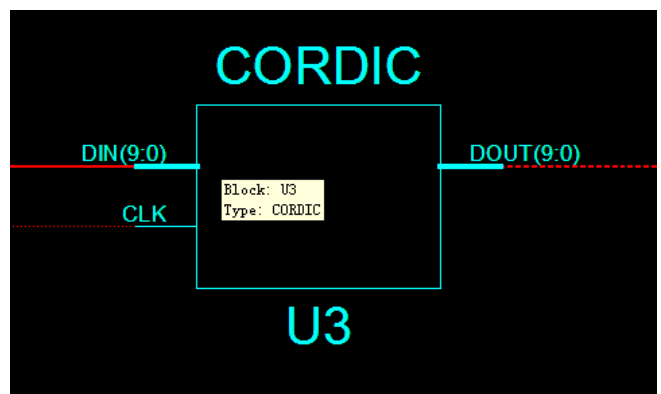

Figure 6. CORDIC IP Core instantiations schematics

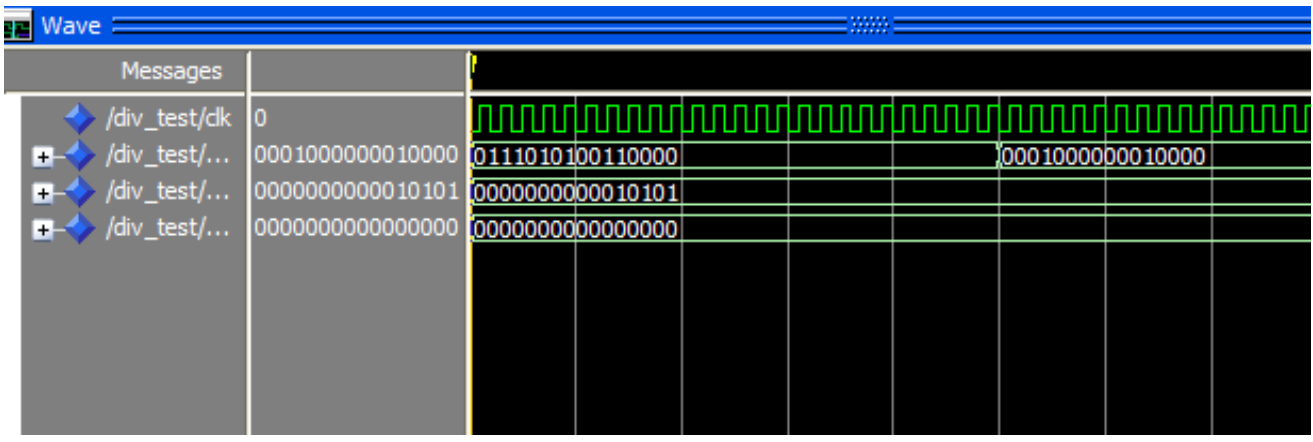

Figure 7. CORDIC functional simulation results

In this paper, a laser range finding system is designed and accomplished. The system has three frequencies $(1 \mathrm{MHz}, 10 \mathrm{MHz}$ and $100 \mathrm{MHz})$ generated by DDS as measurement ruler, the measurement range can be $100 \mathrm{~m}$. In this system, the sampling frequency of $\mathrm{AD}$ is $500 \mathrm{MHz}$. Assuming the phase difference is $30^{\circ}$, calculation of 16 bit word length, echo signal to noise ratio is $12 \mathrm{~dB}$, the average value of multi-measurement phase difference is $30.0217^{\circ}$, and the measurement error is only $0.0217^{\circ}$. Thus, the range finding system has a high performance on phase ranging accuracy, and satisfies the timely measurement as well.

\section{Conclusion}

For the complex of detection algorithm, poor real-time performance, low measuring accuracy in laser range finding system, a multi-frequency phase modulate laser ranging method is developed, design a multi-frequency laser range finding system based on FPGA. This system adopts phase detection algorithm to calculate the sinusoidal value of the measurement distance phase difference under noise environment, and then using CORDIC operator to calculate the measurement distance phase difference. When the sampling frequency is $500 \mathrm{MHz}$, the word length is $16-\mathrm{bits}$, the SNR is $12 \mathrm{~dB}$, the measurement range is $100 \mathrm{~m}$, the phase difference resolution is higher than $0.0217^{\circ}$, and the distance accuracy is $0.12 \mathrm{~mm}$.

\section{Reference}

Chad English, Adam Deslauriers \& Iain Christie. (2005). The complementary nature of triangulation and ladar technologies.Proceedings of SPIE. Laser Radar Technology and Applications X, 29, 27-41. http://dx.doi.org/10.1117/12.603728

Jia, F. X., Ding, Z. L., \& Yuan, F. (2010). Dynamic Target Real-time Laser Ranging System Based On All Phase Fast Fourier Transform Spectrum Analysis. Acta Optica Sinica, 30, 2928-2934. http://dx.doi.org/10.3788/AOS20103010.2928

KOTA, K., \& CAVALLARO, J. R. (1993). Numerical Accuracy and Hardware Tradeoffs for CORDIC Arithmetic for Special-purpose Processor. IEEE Transactions on Computers (Vol. 42, pp. 769-779). http://dx.doi.org/10.1109/12.237718

Li, G. Y., Chen, L., \& Chen, Y. (2011). Research On High Accuracy Multiple Frequency Modulation Laser Ranging Algorithm. Acta Optica Sinica, 40, 1888-1892.

Meher, P. K., Valls, J., \& Tso, B. J. (2009). 50 Years of CORDIC: Algorithms, Architectures, and Applications. Circuits and Systems I: Regular Papers. IEEE Transactions on, 56, 1893-1907. 
Qiu, L., \& Wang, R. L. (2006). Spectrum Analysis Technology of Doppler Ultrasonic Flowmeter. Microcomputer Information, 22, 181-182.

Yang, F., He, Y., \& Chen, W. B. (2008). The Space-borne Laser Coherent Altimeter Parametric Design. Acta Optica Sinica (Vol. 28, pp. 573-577). http://dx.doi.org/10.3788/AOS20082803.0573

Yang, K. (1999). Laser Testing Principle and Technology (pp. 126-135). Wuhan: Huazhong University of Science and Technology Press.

\section{Copyrights}

Copyright for this article is retained by the author(s), with first publication rights granted to the journal.

This is an open-access article distributed under the terms and conditions of the Creative Commons Attribution license (http://creativecommons.org/licenses/by/3.0/). 